\title{
The asymmetric band structure and electrical behavior of the $\mathrm{GdScO}_{3} / \mathrm{GaN}$ system
}

S. lacopetti, P. Shekhter, R. Winter, T. C. U. Tromm, J. Schubert, and M. Eizenberg

Citation: Journal of Applied Physics 121, 205303 (2017); doi: 10.1063/1.4983559

View online: http://dx.doi.org/10.1063/1.4983559

View Table of Contents: http://aip.scitation.org/toc/jap/121/20

Published by the American Institute of Physics

\section{Articles you may be interested in}

Vertical transport in isotype InAIN/GaN dipole induced diodes grown by molecular beam epitaxy

Journal of Applied Physics 121, 205702 (2017); 10.1063/1.4983767

Infrared dielectric functions, phonon modes, and free-charge carrier properties of high-Al-content $\mathrm{Al}_{x} \mathrm{Ga}_{1-\mathrm{x}^{\mathrm{N}}}$ alloys determined by mid infrared spectroscopic ellipsometry and optical Hall effect

Journal of Applied Physics 121, 205701 (2017); 10.1063/1.4983765

A hybrid simulation technique for electrothermal studies of two-dimensional GaN-on-SiC high electron mobility transistors

Journal of Applied Physics 121, 204501 (2017); 10.1063/1.4983761

Precise thickness control in recess etching of $\mathrm{AIGaN} / \mathrm{GaN}$ hetero-structure using photocarrier-regulated electrochemical process

Journal of Applied Physics 121, 184501 (2017); 10.1063/1.4983013

High temperature operation of $n-A I G a N$ channel metal semiconductor field effect transistors on low-defect AIN templates

Applied Physics Letters 110, 193501 (2017); 10.1063/1.4982656

Molecular beam epitaxy of 2D-layered gallium selenide on GaN substrates

Journal of Applied Physics 121, 094302 (2017); 10.1063/1.4977697

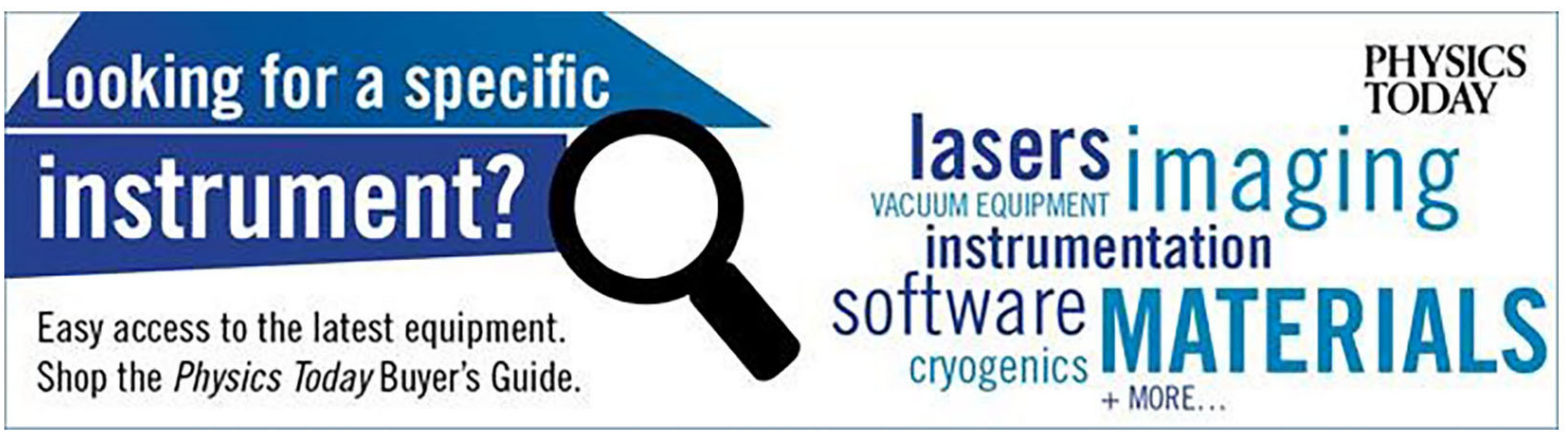




\title{
The asymmetric band structure and electrical behavior of the $\mathrm{GdScO}_{3} / \mathrm{GaN}$ system
}

\author{
S. lacopetti, ${ }^{1, a)}$ P. Shekhter, ${ }^{1}$ R. Winter, ${ }^{1}$ T. C. U. Tromm, ${ }^{2}$ J. Schubert, ${ }^{2}$ and M. Eizenberg ${ }^{1}$ \\ ${ }^{1}$ Department of Materials Science and Engineering, Technion-Israel Institute of Technology, Haifa, Israel \\ ${ }^{2}$ Peter Grünberg Institute 9, Forschungszentrum Jülich GmbH, Jülich, Germany
}

(Received 6 February 2017; accepted 3 May 2017; published online 24 May 2017)

\begin{abstract}
III-V nitrides are interesting materials for a very wide variety of electronic and optoelectronic devices. In this study, their interaction with $\mathrm{GdScO}_{3}$ (GSO), a ternary rare earth oxide, is investigated for MOS applications. We compare pulsed laser deposited amorphous and crystalline epitaxial $\mathrm{GdScO}_{3}$ in terms of their band alignment with the underlying $\mathrm{GaN}$ substrate and the resulting electrical characteristics of the MOS stack. The crystal structure of $\mathrm{GdScO}_{3}$ and $\mathrm{GaN}$ is investigated by means of $\mathrm{x}$-ray diffraction, showing that crystalline oxide is growing epitaxially on GaN. X-ray photoelectron spectroscopy analysis shows a staggered band alignment with a $\mathrm{GdScO}_{3}-\mathrm{GaN}$ valence band offset of $3.6-3.7 \mathrm{eV}$, which is reflected in a very asymmetric current-voltage behaviour of the MOS capacitors: breakdown at positive bias, significantly earlier for the crystalline oxide (around $5 \mathrm{MV} / \mathrm{cm}$ ) compared to the amorphous oxide (around $8 \mathrm{MV} / \mathrm{cm}$ ), and no breakdown up to a field of $-14 \mathrm{MV} / \mathrm{cm}$ at negative bias. Transmission electron microscopy images show a crystalline, two-atom thick interface layer between $\mathrm{GaN}$ and both crystalline and amorphous $\mathrm{GdScO}_{3}$, which is thought to be an electron barrier between GSO and GaN and a possible source of the staggered band alignment. The electrical behaviour can be exploited for asymmetric nano-electronic devices. Published by AIP Publishing.
\end{abstract}

[http://dx.doi.org/10.1063/1.4983559]

\section{INTRODUCTION}

III-V nitrides are characterized by wide, direct band gap and high electron mobility hence, they have been, in recent times, extensively studied for optoelectronic as well as power devices. ${ }^{1,2}$ These semiconductors found a major application in the light emitting diode (LED) industry, where $\mathrm{GaN}$ is used to produce white LEDs, while the research is looking towards UV and deep UV LEDs. ${ }^{3}$ Due to their high and composition-tuneable band gap, ${ }^{4}$ thermal and chemical stability, and high mobility aforementioned, III-V nitrides have also been studied for metal-oxidesemiconductor field effect transistors (MOSFETs), high electron mobility transistors (HEMTs), radio frequency (RF) and microwave devices, sensors, and solar cells, especially for space application where resistance to hard radiation is necessary. ${ }^{5,6}$ One important semiconductor in the III-V nitride family is GaN that shows a direct band gap of $3.4 \mathrm{eV}$ and can be grown on $\mathrm{Si}(111)$ wafers by molecular beam epitaxy (MBE), making it an interesting substitute for $\mathrm{Si}$ - and GaAs-based devices. ${ }^{1}$

High-k oxides have been extensively studied in order to achieve high gate oxide capacitance without decreasing the oxide layer thickness, which would increase the formation of leakage currents. The main requirements for a high-k gate oxide are chemical and structural compatibility with the semiconductor and a wide band gap. ${ }^{7}$ Amorphous $\mathrm{HfO}_{2}$ is already being used as a high-k dielectric deposited on Si-based devices, but it still shows unwanted recrystallization and high diffusivity of oxygen, which induces non-reproducible modification

a)iasara@tx.technion.ac.il of electrical characteristics of the final device; stable single crystals do not recrystallize during processing as well as do not induce surface defects in the semiconductor, and therefore, they are suitable candidates for a reliable gate oxide, as indicated by the ITRS international roadmap of semiconductors. ${ }^{8-10}$ Rare-earth oxides are characterized by a large dielectric constant combined with a relatively wide band gap and chemical stability. Studies on ternary rare-earth oxides showed stable crystallinity and very low oxygen diffusivity, making them interesting candidates for better performances of the dielectric. ${ }^{11}$

In this study, we focused on the electrical and structural characterization of MOS capacitors based on GaN as a semiconductor and $\mathrm{GdScO}_{3}$ (GSO) as a high-k dielectric, as the latter shows a dielectric constant of 24 in the stable hexagonal phase, as discovered in previous studies. ${ }^{12}$ Single crystalline and amorphous GSO were deposited on GaN aiming at a comparison of electrical characteristics and phase stability between single crystalline and amorphous layers. The differences in band alignment and electrical behaviour were investigated for both the amorphous and crystalline oxides deposited on GaN.

\section{EXPERIMENTAL}

A $1 \mu$ m thick $\mathrm{n}$-GaN (Si-doped, $\mathrm{n}=3 \times 10^{18} \mathrm{~cm}^{-3}$ ) layer was grown by MBE on top of $\mathrm{Si}(111)$ with the assistance of buffer layers to minimize the dislocations in the $\mathrm{GaN}$. Crystalline and amorphous GSO were subsequently grown by pulsed laser deposition (PLD). A $\mathrm{KrF}$ excimer laser $(\lambda=248 \mathrm{~nm})$ was used to deposit GSO in an $\mathrm{O}_{2}$ ambient $\left(\mathrm{p}_{\mathrm{O} 2}>10^{-3} \mathrm{mbar}\right.$ ), with a pulse width of $20 \mathrm{~ns}$ and a fluence 
of around $5 \mathrm{~J} / \mathrm{cm}^{2}$ at a repetition rate of $10 \mathrm{~Hz}$. A preheat temperature of $700{ }^{\circ} \mathrm{C}$ was applied for the crystalline GSO deposition, while room temperature was used for the deposition of the amorphous oxide. Two different thicknesses of the oxide, $4 \mathrm{~nm}$ and $30 \mathrm{~nm}$, were chosen according to the required analysis method. A bare GaN sample was used for structural and band alignment characterization.

$\mathrm{Al}$ contacts for MOS capacitors were deposited using an e-gun with a thickness of $100 \mathrm{~nm}$. The substrate (back) contact for each sample was obtained by reactive ion etching (RIE) through the oxide layer using a mixture of $\mathrm{CF}_{4}$ and $\mathrm{O}_{2}$ at $\mathrm{P}<5 \mathrm{mTorr}$, room temperature, and a RF power of $175 \mathrm{~W}$, followed by $100 \mathrm{~nm}$ thick $\mathrm{Al}$ deposition using an e-gun on the exposed GaN.

$\mathrm{X}$-ray diffraction (XRD) and X-ray reflectivity (XRR) measurements were performed in a Rigaku SmartLab X-ray diffractometer equipped with a $\mathrm{Cu} \mathrm{K} \alpha$ source and a rotating anode, making use of a $\mathrm{Ge}(220)$ two-bounce monochromator. $\mathrm{GaN}$ is a wurtzite phase, and on top of it, an epitaxial hexagonal oxide layer was grown, and therefore, $\theta-2 \theta$ spectra were acquired with tilting angles of $0^{\circ}$ [corresponding to the (002) plane] and $61.92^{\circ}$ [corresponding to the (101) plane of GSO]; $\mathrm{d}$-spacings are proportional to $c$ and $a$ as previously derived in Ref. 13. The tilting angle was defined by the measured $c$ lattice constant of GSO and the initial hypothesis of $a / c$ having approximately the value of $a / c$ measured in $\mathrm{GaN}$; repetitions of the experiment for a few angles in the interval of $\pm 1^{\circ}$ around the central value were carried out to remain in agreement with the highest intensity of the signal.

$\mathrm{X}$-ray photoelectron spectroscopy (XPS) analyses were carried out on the $4 \mathrm{~nm}$ thick film and bare samples in order to get signals from both GSO and GaN underneath, hence giving the possibility to determine the band alignments of crystalline and amorphous GSO-GaN. A Thermo VG Scientific Sigma Probe with a monochromatic $\mathrm{Al} \mathrm{K} \alpha$ x-ray source $(\mathrm{h} \nu=1486.6 \mathrm{eV})$ was used for the XPS experiments. Ga $3 p$ and $\mathrm{O} 1 s$ peaks were acquired with a pass energy of $50 \mathrm{eV}$ and fitted by applying Shirley background and Lorentzian to a Gaussian peak ratio of $15 \%$, using the XPSPeak 4.1 software. Charge removal was done by comparison of the Ga3p peak with the values of the same peaks in $\mathrm{GaN}$ standards from the literature,${ }^{14-16}$ aligning the $\mathrm{Ga} 3 p^{3 / 2}$ peak at $105.1 \mathrm{eV}$. The valence band (VB) and electron energy loss (EELS) spectra of the $\mathrm{O} 1 s$ peak have also been acquired with the same resolution of $0.05 \mathrm{eV}$.

Current-voltage (I-V) measurements of MOS capacitors based on the $30 \mathrm{~nm}$ thick oxides were carried out using an Agilent $4155 \mathrm{C}$ tool. A sweep DC voltage was applied in negative bias up to the limit of the instrument $(0,-40 \mathrm{~V})$ and in positive bias up to the breakdown (BD) voltage of the sample. Contact areas were measured using an optical microscope. Transmission electron microscopy (TEM) Bright Field images were acquired using a Tecnai $G^{2}$ T20 S-Twin TEM.

\section{RESULTS AND DISCUSSION}

The $\theta-2 \theta$ spectra of the crystalline $30 \mathrm{~nm}$ thick GSO film, amorphous $30 \mathrm{~nm}$ thick GSO film, and bare GaN sample are presented in Fig. 1. It can be observed that, in addition to the

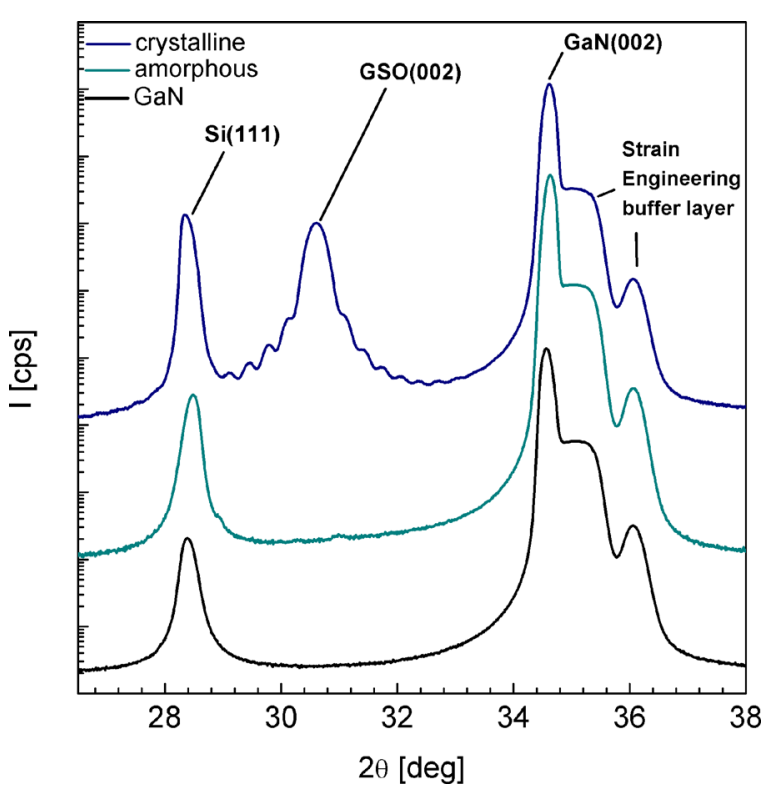

FIG. 1. $\theta-2 \theta$ spectra of crystalline GSO-GaN, amorphous GSO-GaN, and bare $\mathrm{GaN}$.

$\mathrm{Si}(111)$ reflection, two peaks at $2 \theta=30.62^{\circ}$ and $2 \theta=34.60^{\circ}$ are present in the crystalline spectrum. The bare $\mathrm{GaN}$ and the amorphous GSO-GaN show only the $\mathrm{GaN}(002)$ reflection at $2 \theta=34.60^{\circ}$, hence confirming that the first oxide is single crystalline and the second is an amorphous phase. The lattice parameter of the cell is $c_{\text {GaN }}=5.2 \AA$ for GaN in all the cases and $c_{o x}=5.8 \AA$ for GSO in the case of the crystalline oxide. A shoulder of the GaN peak at higher angles is visible in all the spectra: its multi-peak shape is compatible with the strain engineering buffer layer deposited on $\mathrm{Si}(111)$ before the deposition of GaN by MBE, as mentioned in the fabrication procedure.

A $\theta-2 \theta$ scan with a tilt angle of $\chi=61.37^{\circ}$ on the crystalline $30 \mathrm{~nm}$ GSO film exhibits a peak from the (101) plane of GSO at $2 \theta=31.98^{\circ}$ while $\mathrm{GaN}$ produced a peak at $2 \theta$ $=36.49^{\circ}$, associated with the values of $a_{o x}=3.7 \AA$ and $a_{G a N}$ $=3.2 \AA$ (Fig. 2), corresponding to a lattice mismatch of

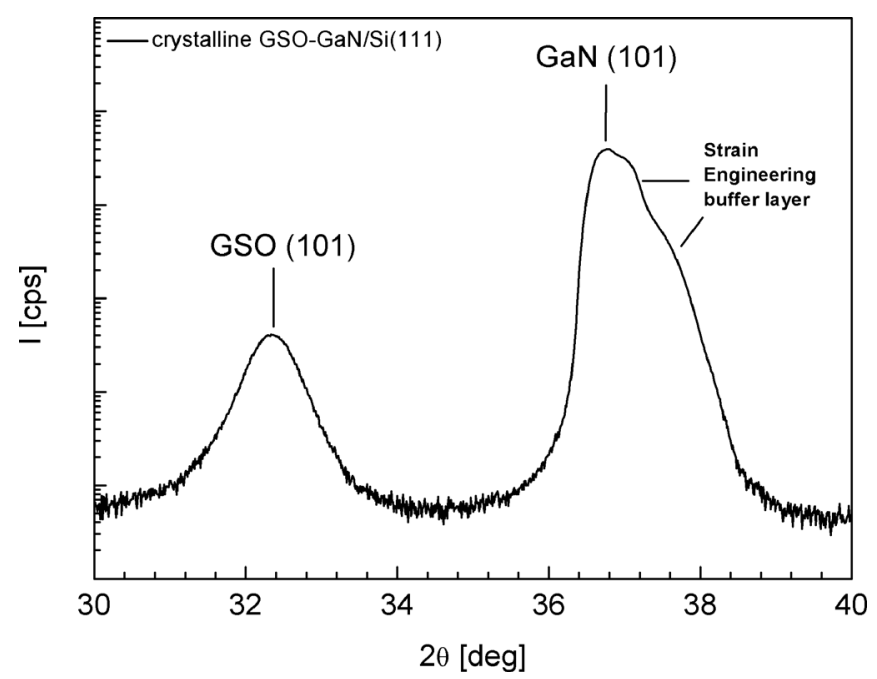

FIG. 2. Tilted $\theta-2 \theta$ spectrum of crystalline GSO-GaN/Si, showing reflection of the (101) plane from GaN and GSO. 


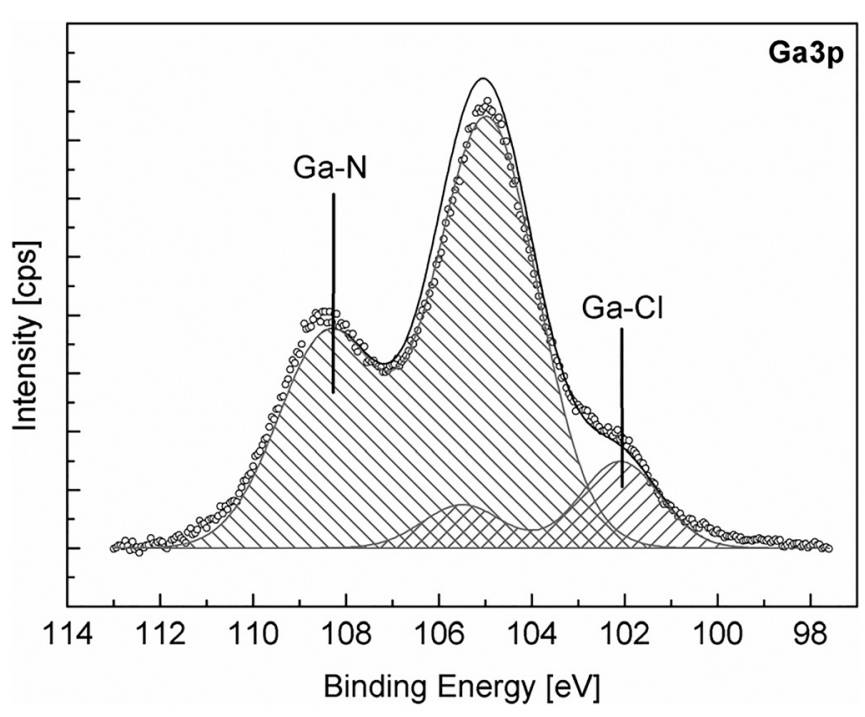

FIG. 3. Ga3p peak of the bare $\mathrm{GaN}$ substrate, showing $\mathrm{Cl}$ contamination and no native oxide formation.

$\sim 14 \%$, coherent with the values found in the literature. ${ }^{12}$ Therefore, a hexagonal relaxed epitaxial GSO layer has been successfully grown on $\mathrm{GaN}$ (wurtzite).

The exact thickness of these oxides was verified by the XRR technique (spectra not shown), yielding values of $27.9 \mathrm{~nm}$ and $28.0 \mathrm{~nm}$ for the crystalline oxide and the amorphous, respectively.

XPS analysis shows chlorine contamination in GaN (Fig. 3): this is due to commonly used cleaning procedures. ${ }^{17}$ Band alignment was determined by finding, via XPS measurements, the $\mathrm{E}_{\text {gap }}$ of GSO and the valence band offset (VBO) of $\mathrm{GaN}$ with respect to $\mathrm{GSO} .{ }^{18} \mathrm{E}_{\text {gap }}=3.4 \mathrm{eV}$ for wurtzite $\mathrm{GaN}$ was used. ${ }^{4}$ Electron energy loss spectroscopy (EELS) of the Ols peak was used to evaluate $\mathrm{E}_{\text {gap }}$ of GSO (Fig. 4). The values found in this way are $5.3 \pm 0.1 \mathrm{eV}$ and $5.4 \pm 0.1 \mathrm{eV}$ for the crystalline and amorphous GSO, respectively, in agreement with the values found in the literature. ${ }^{11,12}$ In the

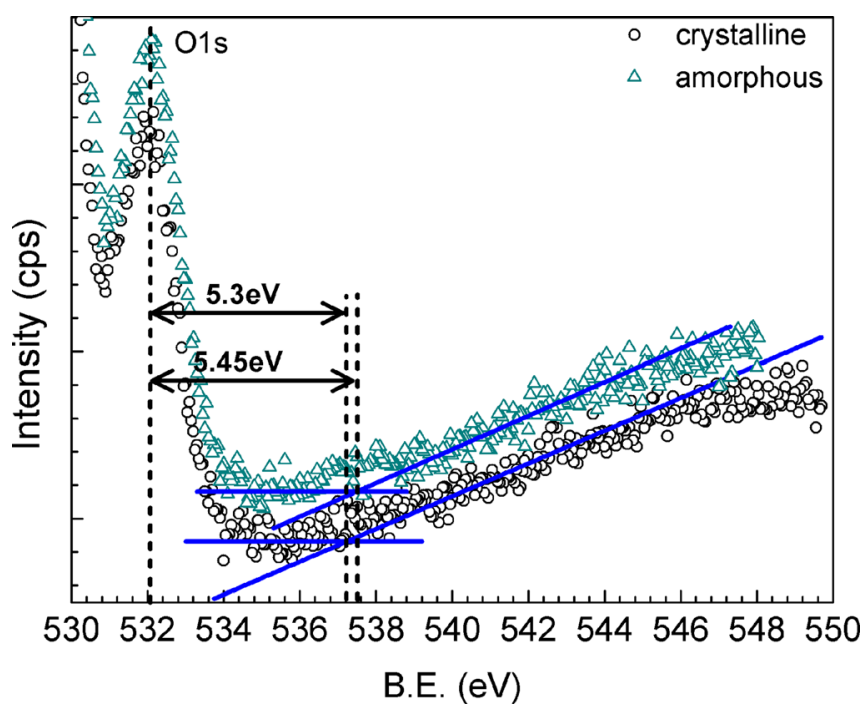

FIG. 4. EELS and energy gap of crystalline GSO-GaN and amorphous GSO-GaN. In blue, peak linear regression and background average are higlighted.

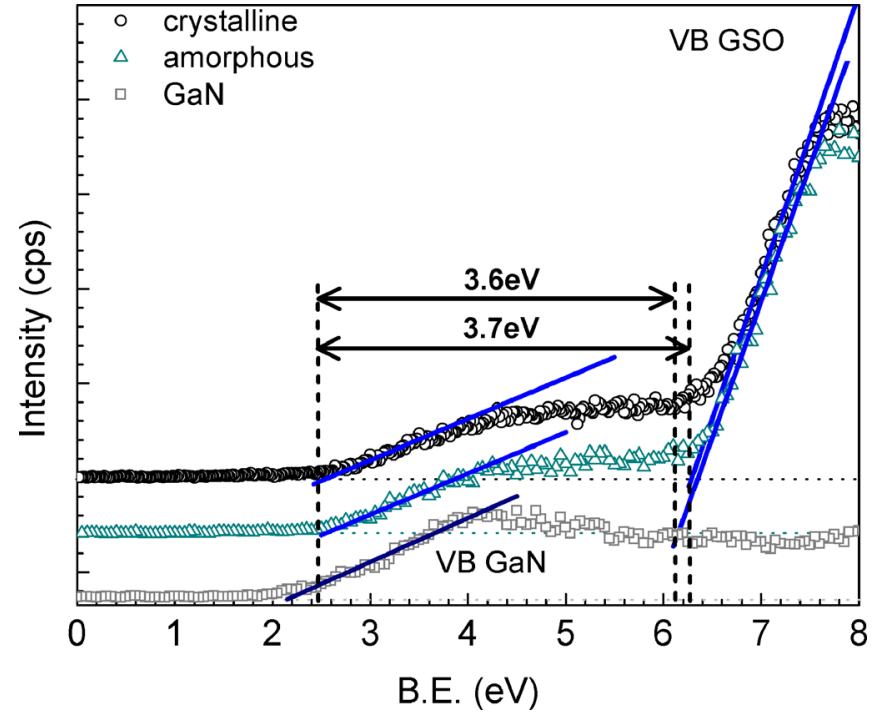

FIG. 5. VBO calculated from the VB spectra of crystalline GSO-GaN, amorphous GSO-GaN, and bare GaN. In blue, VB linear regressions are highlighted.

systems under analysis, electrons are emitted from both GSO and GaN since the oxide film thickness is lower than the escape depth of the electrons in the operational conditions, namely 3 times the incident wavelength. Hence, the two onsets of VB are visible at low binding energies (Fig. 5). The distinction between the VB of GSO and GaN has been deduced from the analysis of the bare $\mathrm{GaN}$ sample. The VBO of GaN with respect to that of GSO is calculated from the contributions in the VB region in the XPS spectra (Fig. 5), and its values are $3.7 \pm 0.1 \mathrm{eV}$ and $3.6 \pm 0.1 \mathrm{eV}$ for the crystalline and the amorphous oxides, respectively. The band alignment of the two systems is summarised in Fig. 6.

It can be seen that the band alignment of the two systems is practically identical with a negative conduction band offset (CBO) of $1.6-1.8 \mathrm{eV}$ between $\mathrm{GaN}$ and GSO, which means that the band alignment is staggered, an unlikely situation when high- $\kappa$ oxides are deposited on top of semiconductors, according to what is found in the literature. $^{11,19}$

The staggered band alignment is expected to have a strong influence on the electrical behaviour of the $\mathrm{Al} / \mathrm{GSO} /$ GaN MOS capacitors since no potential barrier exists for electrons in $\mathrm{GaN}$ when applying a positive bias to the gate

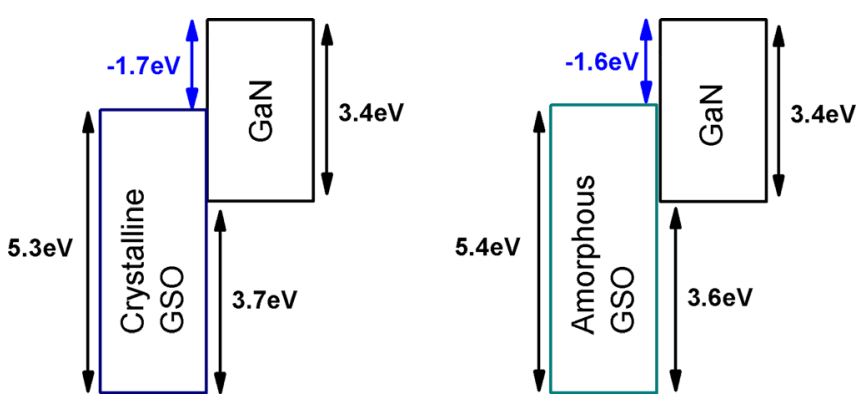

FIG. 6. Band alignment of both amorphous and crystalline GSO on GaN. In blue, the negative conduction band offset is highlighted. 
electrode. At negative bias, a large barrier for holes exists, as well as a very high barrier for electrons. This hinders any significant current up to higher fields than in the case of the straddling band alignment which would give an asymmetry for $\mathrm{n}$-doping only. The current-voltage (IV) behaviour was analysed (Fig. 7) in light of the asymmetric band alignment. JE curves are plotted to show current values normalised to the capacitors' area and applied voltage values normalised to the film thickness. The breakdown (BD) for the crystalline GSO film with applied positive bias is always detected around fields of $(+6 \pm 1.5) \mathrm{MV} / \mathrm{cm}$, while the breakdown has never been observed at fields less negative than $-14 \mathrm{MV} / \mathrm{cm}$ when applying negative bias. In positive bias, a gradual increase in current is noticeable prior to $\mathrm{BD}$.

$\mathrm{Al} / \mathrm{amorphous} \mathrm{GSO} / \mathrm{GaN}$ capacitors produce breakdown in forward bias at statistically higher electric fields than the crystalline GSO/GaN in the same conditions, between 8 and $9 \mathrm{MV} / \mathrm{cm}$. The same behaviour of the crystalline GSO was observed when applying negative bias. The asymmetric behaviour is clearly marked, coherently with the staggered band alignment found via XPS analysis of the $4 \mathrm{~nm}$ film sample; nonetheless, the breakdown at positive bias is not immediate. The higher BD voltage in the case of the amorphous oxide can be inferred by the higher resistance of the amorphous film with respect to the crystalline film or by the higher defectiveness of the crystalline film induced by defects of the substrate on which it was epitaxially grown. It can be hypothesised that GaN dislocations, that cause large pinholes in $\mathrm{GaN},{ }^{1}$ propagate into the crystalline GSO layer and create paths of least resistance that give way to earlier breakdown. The amorphous layer, however, is not sensitive to GaN defects as it does not reproduce the crystallinity of the substrate. In addition to these considerations, the hypothesis of the presence of an interfacial electron barrier not detected by XPS should be strongly considered.

TEM images of crystalline and amorphous GSO-GaN interfaces are given in Figs. 8 and 9, respectively. An

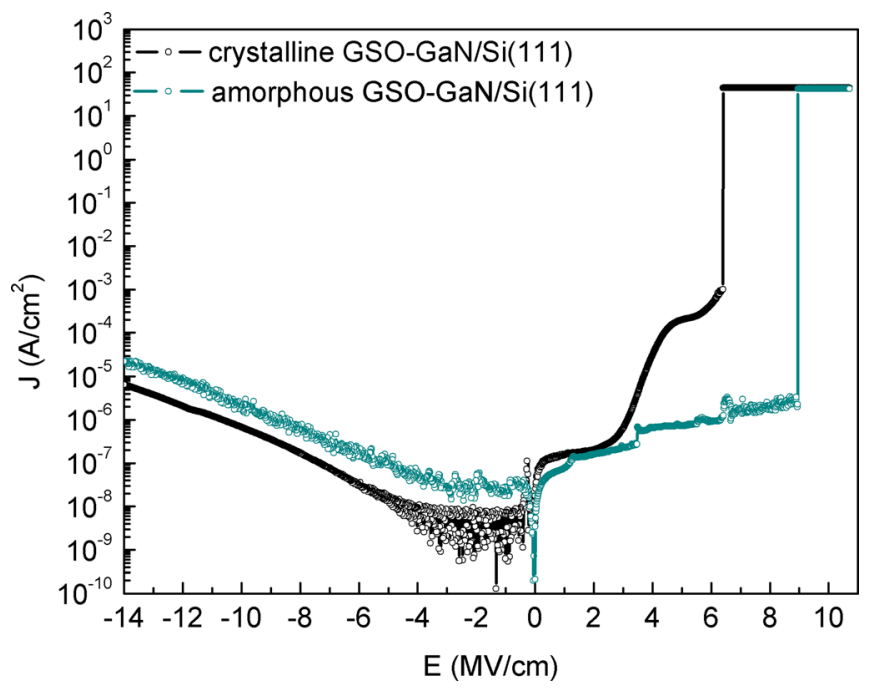

FIG. 7. JE plot of crystalline GSO-GaN and amorphous GSO-GaN. Asymmetric positive-negative electrical behaviour observed due to $\mathrm{BD}$ voltage in positive bias.

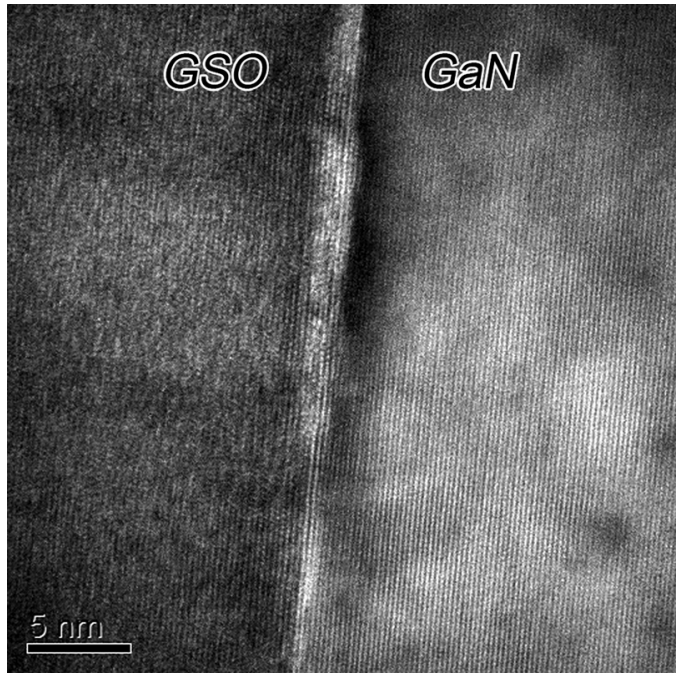

FIG. 8. TEM image of crystalline GSO-GaN showing the nature of the interface layer.

interface layer can be noticed between GaN and GSO. In bright field TEM, it appears with a lighter contrast, indicating a lower density. Unlike spontaneous interfacial layers, the layer observed appears to have a crystalline nature, pointing to the fact that it is not a native oxide but rather a part of the deposited film. This observation, combined with the absence of $\mathrm{Ga}-\mathrm{O}$ bonds in the $\mathrm{Ga} 3 p$ spectrum reported in Fig. 3 and no $\mathrm{N}$ bonds other than $\mathrm{N}-\mathrm{Ga}$ detected in the $\mathrm{N} / s$ peak (not shown), leaves the possibility of a $\mathrm{Sc}_{2} \mathrm{O}_{3}$ segregation at the interface occurring during or after the PLD deposition. According to the literature, binary rare earth oxides such as $\mathrm{Sc}_{2} \mathrm{O}_{3}$ and $\mathrm{La}_{2} \mathrm{O}_{3}$ show a positive conduction band offset. ${ }^{11,19}$ It can be hypothesised that the so-called Sc-rich layer constitutes a thin electron barrier between the semiconductor and the dielectric, representing the source of the structure's band alignment and asymmetric electrical behaviour with non-immediate breakdown at positive bias. Thereby, further studies on the full structure's band alignment are currently carried out.

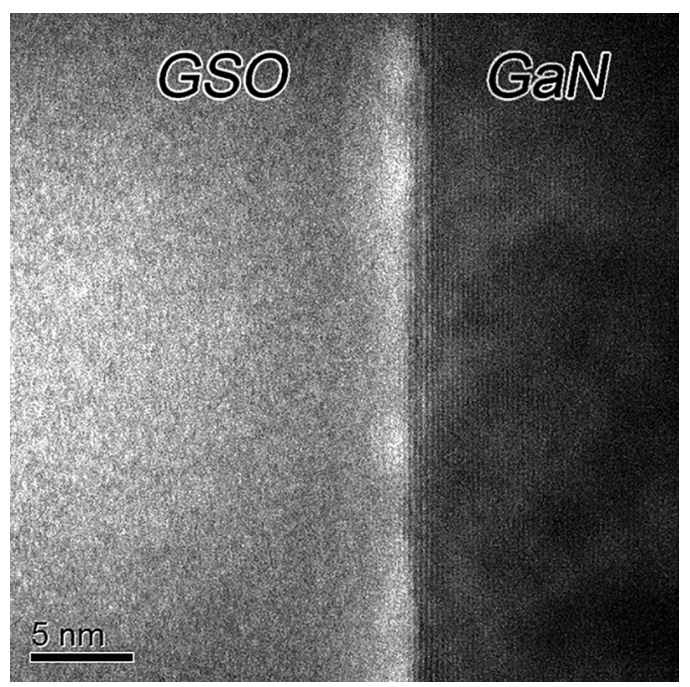

FIG. 9. TEM image of amorphous GSO-GaN showing the crystalline interface layer. 


\section{SUMMARY}

The system of GSO deposited on top of GaN was characterised structurally and electrically. XRD analyses show that single crystalline GSO is successfully grown as a hexagonal layer on top of wurtzite $\mathrm{GaN}$, with a lattice bigger by $14 \%$. Amorphous GSO is successfully obtained, without any GSO crystallization taking place. The band alignment of GSO and GaN was found to be staggered, with no conduction band barrier for both the crystalline and amorphous oxides. Due to the absence of a barrier for electrons, the electrical behaviour of these systems became extremely asymmetric with different current characteristics on either bias. $\mathrm{BD}$ was recorded only at positive bias with an average field of around $5 \mathrm{MV} / \mathrm{cm}$ for the epitaxial oxide, while fields of up to $14 \mathrm{MV} / \mathrm{cm}$ did not cause any $\mathrm{BD}$ on the negative side. It was also seen that the amorphous GSO film is more resilient against BD than its crystalline counterpart. A thin crystalline $\mathrm{Sc}_{2} \mathrm{O}_{3}$ segregation layer was visible at both the crystalline and amorphous GSO-GaN interfaces, and it is believed to be a thin electron barrier between $\mathrm{GaN}$ and GSO, responsible for the non-immediate BD at positive bias. The asymmetric electrical behaviour can be utilised to fabricate asymmetric nano-electronic devices, while the staggered band alignment results in a disadvantage for the high- $\kappa$ oxide application in nitride-based MOS devices, original intention of the investigation. However, the presence of a Sc-rich electron barrier between GSO and GaN, if confirmed in further experiments, will allow the application of these systems in GaN-based MOS devices, with room for investigation on the nature and role of the barrier layer as a function of production parameters and treatments.

\section{ACKNOWLEDGMENTS}

The authors thank the German-Israeli Foundation for Scientific Research and Development for financial support of this Project (I-1273-401.10/2014).

${ }^{1}$ F. Medjdoub and K. Iniewski, Gallium Nitride (GaN): Physics, Devices, and Technology, 1st ed. (Taylor \& Francis Group, LLC, 2016).

${ }^{2}$ V. R. Reddy, M. S. P. Reddy, B. P. Lakshmi, and A. A. Kumar, J. Alloys Compd. 509, 8001 (2011).

${ }^{3}$ J. W. Orton and C. T. Foxon, Rep. Prog. Phys. 61, 1 (1998).

${ }^{4}$ E. F. Schubert, T. Gessmann, and J. K. Kim, in Kirk-Othmer Encyclopedia of Chemical Technology (John Wiley \& Sons, Inc., Hoboken, NJ, USA, 2005).

${ }^{5}$ R. F. Davis, Proc. IEEE 79, 702 (1991).

${ }^{6}$ S. J. Pearton, F. Ren, E. Patrick, M. E. Law, and A. Y. Polyakov, ECS J. Solid State Sci. Technol. 5, Q35 (2016).

${ }^{7}$ J. Robertson and R. M. Wallace, Mater. Sci. Eng. R Rep. 88, 1 (2015).

${ }^{8}$ M. Fanciulli and G. Scarel, Rare Earth Oxide Thin Films (Springer, Berlin, Heidelberg, 2006).

${ }^{9}$ D. J. Lichtenwalner, in High Permittivity Gate Dielectric Materials, edited by in S. Kar (Springer Berlin Heidelberg, 2013).

${ }^{10}$ R. Harper, Mater. Sci. Eng. B Solid-State Mater. Adv. Technol. 134, 154 (2006).

${ }^{11}$ V. V. Afanas'ev, A. Stesmans, C. Zhao, M. Caymax, T. Heeg, J. Schubert, Y. Jia, D. G. Schlom, and G. Lucovsky, Appl. Phys. Lett. 85, 5917 (2004).

${ }^{12}$ A. Schaefer, A. Besmehn, M. Luysberg, A. Winden, T. Stoica, M. Schnee, W. Zander, G. Niu, T. Schroeder, S. Mantl, H. Hardtdegen, M. Mikulics, and J. Schubert, Semicond. Sci. Technol. 29, 75005 (2014).

${ }^{13}$ W.-S. Han, S.-O. Kang, and I.-H. Su, Korean J. Crystallogr. 18, 7 (2007).

${ }^{14}$ J. Hedman and N. Martensson, Phys. Scr. 22, 176 (1980).

${ }^{15}$ R. Carin, J. P. Deville, and J. Werckmann, Surf. Interface Anal. 16, 65 (1990).

${ }^{16}$ G. Martin, A. Botchkarev, A. Rockett, and H. Morkoç, Appl. Phys. Lett. 2541, 2541 (1995)

${ }^{17}$ S. W. King, J. P. Barnak, M. D. Bremser, K. M. Tracy, C. Ronning, R. F. Davis, and R. J. Nemanich, J. Appl. Phys. 84, 5248 (1998).

${ }^{18}$ S. Miyazaki, Appl. Surf. Sci. 190, 66 (2002).

${ }^{19}$ J. F. Ihlefeld, M. Brumbach, and S. Atcitty, Appl. Phys. Lett. 102, 162903 (2013). 